\title{
Outcomes Following Interval Debulking Surgery in Primary Peritoneal Carcinoma
}

\author{
CAROLINE F. CONNOLLY ${ }^{1}$, SUNDUS YAHYA ${ }^{2}$, KIONG KONG CHAN ${ }^{3}$, KAVITA SINGH $^{3}$, \\ SUDHA SUNDAR $^{3,4}$, SUHAIL M. ANWAR ${ }^{2}$ and INDRAJIT FERNANDO ${ }^{2}$ \\ ${ }^{1}$ University Hospitals of North Midlands NHS Trust, Stoke On Trent, U.K.; \\ ${ }^{2}$ The Cancer Centre, Queen Elizabeth Hospital, Birmingham, U.K.; \\ ${ }^{3}$ Pan-Birmingham Gynaecology Cancer Centre, City Hospital, Birmingham, U.K.; \\ ${ }^{4}$ Institute of Cancer and genomics sciences, University of Birmingham, Birmingham, U.K.
}

\begin{abstract}
Aim: Primary peritoneal carcinoma (PPC) has a poor prognosis, with a median survival of 11-24 months. Unlike ovarian cancer, there exist no published data on the effectiveness of interval debulking surgery (IDS) in PPC and it is not routine practice. Our series compared outcomes in patients with PPC treated with IDS following chemotherapy versus patients treated with chemotherapy alone. Patients and Methods: A retrospective case-note analysis was undertaken of all patients diagnosed with PPC in the PanBirmingham network between May 2000 and October 2008. Data were analysed for age, performance status, response to chemotherapy, surgical outcomes, subsequent treatments, site of relapse, median progression-free (PFS) and overall (OS) survival. Analysis for PFS and OS was undertaken using both Kaplan-Meier and log-rank analysis. Results: A total of 44 patients with histologically-proven PPC were identified: 41 patients received chemotherapy with platinum combination or alone; $17 / 44$ (39\%) of patients underwent IDS following chemotherapy and 15 of these had optimal debulking; $3 / 15$ (20\%) had a complete pathological response. The recurrence rate for the surgical group was $11 / 17$ (65\%) including those with suboptimal debulking, whereas disease recurred in 25/27(93\%) of the non-surgical group. The median PFS was 25 months (range=8-33 months) in the IDS group compared to 9 months (range=0-30 months) in the non-surgical group $(p=0.001)$. The median OS was 48 months in the IDS group compared to 18 months in the non-surgical group $(p=0.0016)$. The median OS for the
\end{abstract}

Correspondence to: Sundus Yahya, c/o Julie Chapman, 3rd Floor Cancer Centre, Queen Elizabeth Hospital, University Hospital Birmingham, B15 2TH, U.K. Tel: +44 1213713597, e-mail: drsundusyahya@yahoo.co.uk

Key Words: Primary peritoneal carcinoma, interval debulking surgery, chemotherapy. whole patient cohort was 32 months. Conclusion: The median OS for the whole cohort compares favourably with previously published survival data of 11-24 months. IDS in selected cases of PPC appears to improve median $O S$ and PFS in PPC and we would recommend that surgery is considered as a treatment option in all patients who have a good response to chemotherapy or entry into a clinical trial.

Primary peritoneal carcinoma (PPC) is a rare intra-abdominal malignancy characterised by peritoneal carcinomatosis with minimal or no involvement of the ovaries. It was first described in 1959 by Swerdlow et al. (1) and has a similar clinical presentation to advanced epithelial ovarian cancer (EOC). The updated International Federation of Gynecology and Obstetrics (FIGO) classification of 2014 has uniformly classified PPC. The histological sub-types described include high-grade serous, low-grade serous, mucinous, clear cell and endometrioid carcinomas (2). A dualistic model of ovarian carcinogenesis has been proposed with type I tumours being low-grade and type II tumours comprising high-grade histology with $p 53$ mutations mostly driving the high-grade histology $(3,4)$. In published data, PPC accounts for $8-15 \%$ of EOC, with PPC having poorer prognosis as compared to other histological subtypes (5-8).

PPC is typically a diagnosis of exclusion after operative and pathological assessment of the ovaries. The Gynaecologic Oncology Group (GOG) developed diagnostic criteria to distinguish PPC from serous EOC (9). These criteria have also been adopted by the World Health Organisation (WHO).

Historically, the prognosis for PPC is inferior to equivalent stage EOC, with a median overall survival of 11.3-24 months $(6,7,10-14)$. There is a lack of randomised data regarding the optimum management of PPC, and it is not commonly distinguished as a distinct clinical entity for the purpose of clinical trial inclusion.

Interval debulking surgery has been used successfully in advanced-stage EOC where tumour bulk has precluded 
maximal primary debulking surgery. The EORTC trial revealed that neoadjuvant chemotherapy followed by interval debulking surgery results in the same survival, but fewer postoperative complications, as primary debulking surgery (8).

The purpose of the present study was to investigate the role of interval debulking surgery in PPC where the advanced nature of the disease usually precludes maximal primary debulking surgery.

\section{Patients and Methods}

All women diagnosed with PPC at four Institutions in the PanBirmingham network between May 2000 and October 2008 were identified retrospectively from oncological records. Patients were excluded if there was any evidence of ovarian masses radiologically, intraoperatively or histologically.

The primary outcome measure for this series was patient survival. Secondary outcome measures included progression-free survival, site of recurrence, response to chemotherapy and the degree of surgical debulking. In addition, baseline patient demographics and performance status, histological subtype, FIGO stage and chemotherapy received were recorded

Chemotherapy. All patients receiving chemotherapy had a platinumbased regimen. These were, single-agent carboplatin at area under the curve (AUC) of 5 or 6 (depending on performance status) or a combination regimen of either carboplatin (AUC5/6)/paclitaxel (175 $\mathrm{mg} / \mathrm{m}^{2}$ ) or carboplatin (AUC5)/ gemcitabine $\left(1250 \mathrm{mg} / \mathrm{m}^{2}\right)$. Three patients did not receive chemotherapy because of poor performance status at diagnosis.

Surgery. All surgery was carried out at the regional gynaecological cancer centre and entailed total abdominal hysterectomy, bilateral salpingo-oophrectomy, omentectomy, lymphaedenectomy and removal of all other macroscopic disease where possible (including bowel resection where indicated).

Evaluation and follow-up. Data were collected until September 2010. The median follow-up was 25.5 (range=1-50) months. Progression or recurrence of disease was defined as new or progressive disease on imaging according to WHO Response Evaluation Criteria in Solid Tumours (RECIST) or an incremental rise in cancer antigen 125 (CA125) by a factor of two compared to nadir (according to GOG criteria) (15). Survival was calculated from the month of diagnosis to the month of death

Statistical analysis. Recurrence-free and overall survival rates were determined by using the Kaplan-Meier method and differences in survival curves were compared using log-rank methods. Hazard ratios were generated through Cox regression analysis. Differences between patient characteristics were analysed using the chi-squared and Fisher's exact tests as appropriate.

Overall survival (OS) was defined from the date of diagnosis to the date of death from any cause. Patients alive or lost to follow-up at the time of analysis were censored at the date last known to be alive). Progression-free survival (PFS) was defined from the date of diagnosis to the earliest date of progression or death. Patients alive and progression-free or lost to follow-up at the time of analysis were censored at the date last known to be progression-free.

\section{Results}

Characteristics of the patients. Between May 2000 and October 2008, 44 patients were identified as having a diagnosis of primary peritoneal carcinoma by radiological imaging and histology. Patient and tumour characteristics and biopsy methods (Table I) showed no significant differences between the two patient groups.

Chemotherapy. The majority of patients in the surgery group $(11 / 17,65 \%)$ received combination chemotherapy compared to $40 \%$ in the non-surgical group, although this was not statistically significant $(p=0.215)$. The median total number of cycles of chemotherapy in the surgical group was 8 (range $=4-8$ ) compared to 6 (range $=0-8$ ) in the non-surgical group. Thirteen patients had split chemotherapy, with 3-6 cycles of induction chemotherapy followed by surgery and further cycles of chemotherapy postoperatively. The total number of cycles received in the surgical group was significantly higher $(p<0.001)$.

Surgery. Seventeen patients underwent interval debulking surgery, out of whom 16 showed a response to chemotherapy with a radiological response according to RECIST (partial or complete response). One patient proceeded to surgery with stable disease. All cases were discussed in a regional multidisciplinary team meeting prior to a surgical decision being made.

Optimal surgical resection (defined as residual disease of $<1 \mathrm{~cm}$ ) was achieved in 15 out of the 17 patients. Three patients were found to have had a complete pathological response with no viable tumour identifiable in the surgical specimen. No patients died within 30 days of surgery.

Details regarding chemotherapy regimen, number of cycles, tumour response, postoperative histology and site of relapse, where applicable, are shown in Table II.

Further treatments. Thirty-two patients had further courses of chemotherapy following relapse of disease: 15 patients in the surgical arm and 17 in the non-surgical arm. Patients were re-challenged with platinum regimens if their disease relapsed after 6 months. In patients deemed to have platinum-resistant disease, other regimens used included liposomal doxorubicin, topotecan and etoposide. Two patients were entered into clinical trials.

Relapse. Up to and including September 2010, disease had relapsed in $11 / 17(65 \%)$ patients in the surgical group compared to $25 / 27$ (93\%) patients in the non-surgical group. Debulking surgery did not significantly influence the site of relapse $(p=0.058)$, with the majority of cases relapsing in the abdomen with peritoneal disease and ascites in both groups. 
Table I. Comparison of patient characteristics, tumour characteristics and biopsy methods for surgical and non-surgical patients.

\begin{tabular}{lcc}
\hline Characteristic & $\begin{array}{c}\text { Interval surgery } \\
(\mathrm{n}=17)(\%)^{*}\end{array}$ & $\begin{array}{c}\text { No surgery } \\
(\mathrm{n}=27)(\%)^{*}\end{array}$ \\
\hline Age, years & & \\
$\quad$ Mean & 68 & 66 \\
Range & $54-82$ & $46-79$ \\
WHO PS & & \\
0 & 0 & 1 \\
1 & $11(65)$ & $12(48)$ \\
2 & $5(29)$ & $12(44)$ \\
3 & $1(6)$ & $2(7)$ \\
4 & 0 & 0 \\
Histology & $13(76)$ & $20(74)$ \\
Serous & $4(24)$ & $7(26)$ \\
Other & & \\
Stage & $11(65)$ & $17(63)$ \\
IIIC & $6(35)$ & $10(37)$ \\
IV & $11(65)$ & $19(70)$ \\
Malignant pleural effusion & $6(35)$ & $8(30)$ \\
No & & \\
Yes & $2(12)$ & $8(30)$ \\
Method of biopsy & $5(29)$ & $8(30)$ \\
Laparotomy & $7(41)$ & $5(19)$ \\
Laparoscopy & $2(12)$ & $3(11)$ \\
Image guidance & $1(6)$ & $3(11)$ \\
Cytology & & \\
Unknown & 647 & 613 \\
Serum CA125 (U/ml) at diagnosis & $134-11,070$ & $21-32,000$ \\
Mean & & \\
Range & & \\
\hline
\end{tabular}

*All percentages rounded to the nearest whole number. CA125: Cancer antigen 125; WHO PS: World Health Organisation performance status.

Overall survival and time to progression. The median overall survival was 48 months in the surgical group and 18 months in the non-surgical group $(p=0.0016)$. The hazard ratio for death in the surgical group compared to the non-surgical group was 0.2947 (95\% confidence interval $=0.1422-0.610$ ) (Figure 1). The median progression-free survival for the surgical group was 25 (range $=8-33$ ) months compared to 9 (range $=0$-30) months for the non-surgical group ( $p=0.001)$ (Figure 2). The median survival for the whole group was 32 months (Figure 3).

\section{Discussion}

PPC and fallopian tube cancer have historically been incorporated into the inclusion criteria for many of the advanced ovarian cancer trials. Uncertainty still exists in the literature as to whether these types of cancer are part of the same disease entity. As PPC only accounts for $8-15 \%$ of EOC (5-8), published data are most often limited to small patient series of fewer than 50 patients.
Table II. Comparison of chemotherapy regimens, number of cycles, tumour response, postoperative histology and site of relapse for surgical and non-surgical patients.

\begin{tabular}{|c|c|c|}
\hline Characteristic & $\begin{array}{l}\text { Interval surgery } \\
\quad(\mathrm{n}=17)(\%)^{*}\end{array}$ & $\begin{array}{l}\text { No surgery } \\
(\mathrm{n}=27)(\%)^{*}\end{array}$ \\
\hline \multicolumn{3}{|l|}{ Chemotherapy } \\
\hline Carboplatin & $6(35)$ & $13(48)$ \\
\hline Carboplatin/taxol & $11(65)$ & $9(33)$ \\
\hline Carboplatin/gemcitabine & $0(0)$ & $2(7)$ \\
\hline None & $0(0)$ & $3(11)$ \\
\hline \multicolumn{3}{|l|}{ Response to chemotherapy } \\
\hline Complete response & $3(18)$ & $1(4)^{* *}$ \\
\hline Partial response & $13(76)$ & $16(67)^{* *}$ \\
\hline Stable disease & $1(6)$ & $1(4)^{* *}$ \\
\hline Progressive disease & $0(0)$ & $6(25)^{* *}$ \\
\hline N/A & $0(0)$ & $3(-)$ \\
\hline No of cycles & Total & Total \\
\hline 0 & 0 & 3 \\
\hline 1 & 0 & 0 \\
\hline 2 & 0 & 1 \\
\hline 3 & 0 & 0 \\
\hline 4 & 0 & 5 \\
\hline 5 & 2 & 1 \\
\hline 6 & 6 & 16 \\
\hline$>6$ & 9 & 1 \\
\hline \multicolumn{3}{|l|}{ Postoperative histology } \\
\hline Microscopic deposits & $7(41)$ & - \\
\hline Macroscopic deposits & $5(29)$ & - \\
\hline Complete pathological response & $3(18)$ & - \\
\hline Unknown & $2(12)$ & - \\
\hline \multicolumn{3}{|l|}{ Site of relapse } \\
\hline Abdomino-peritoneal & 11 & 22 \\
\hline Pleural effusion & 0 & 1 \\
\hline Unknown & 0 & 2 \\
\hline No relapse & 6 & 2 \\
\hline
\end{tabular}

N/A: Not available. *All percentages rounded to the nearest whole number **percentages given for 24 patients only as three patients in this group did not receive chemotherapy.

Complete resection of all macroscopic disease during cytoreductive surgery has been shown to be the single most important prognostic factor in advanced ovarian carcinoma. It is uncertain whether primary cytoreductive surgery is of value in treatment for advanced disease, such as in PPC, where complete removal of macroscopic disease is not possible due to disease extent. The recent EORTC trial confirmed the value of interval debulking surgery in advanced ovarian cancer (8). This study included 48 patients with PPC, although no subset analysis was performed on this group.

PPC is of multifocal, multiclonal origin and often infiltrates the peritoneum, liver, spleen and mesentry such that optimum cytoreduction is not possible upfront. Barda $e t$ al. showed that compared to EOC-matched controls, those with PPC did not show as much benefit from optimum 


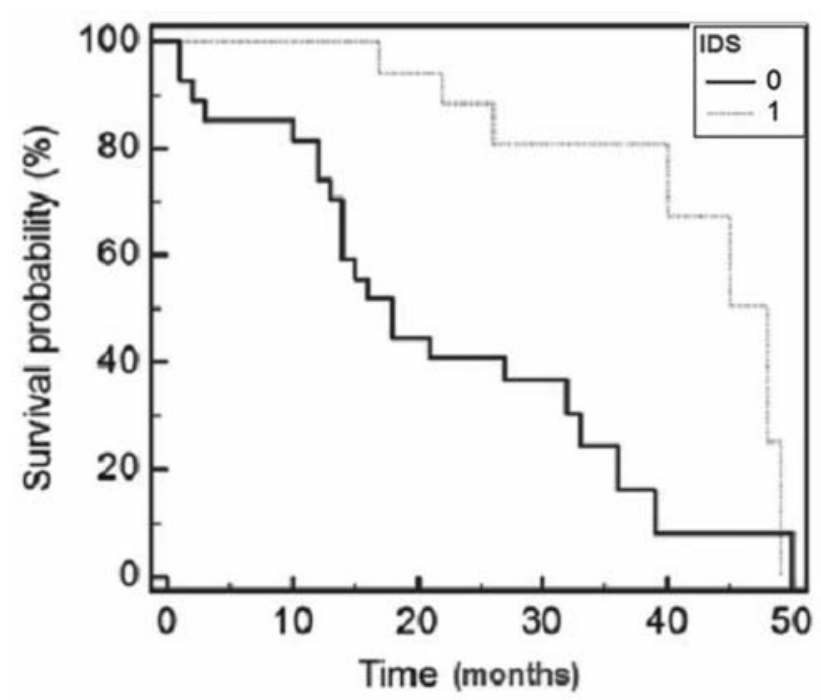

Figure 1. Kaplan-Meier curve comparing overall survival of surgical (1) and non-surgical (0) patients $(p=0.0016)$. Hazard ratio $=0.2947(95 \%$ confidence interval=0.1422-0.610). IDS: Interval debulking surgery.

cytoreductive surgery and the diameter of residual disease did not have an impact on survival (16).

Furthermore, the average age at diagnosis is significantly older in PPC (3) than comparative stage EOC. This may also affect a clinician's willingness to consider the patient as a surgical candidate.

Our series correlates well with current data in advanced EOC (17). If optimal cytoreductive surgery is achieved, following induction platinum-based chemotherapy, patients with PPC have significantly improved overall and progression-free survival.

Clearly in our non-randomised patient series, selection bias exists, with those achieving a good response to chemotherapy and maintaining a good performance status being selected for surgery. A higher percentage of patients in the surgical group received combination chemotherapy, which reflects their better performance status. However, data from ICON3 (18) and NeoEscape (19) trials show there is no survival difference with single-agent carboplatin compared with combinations regimens. It should be noted that the median survival of the whole group compares favourably with previously published survival data, suggesting the results cannot be explained by selection bias alone. The results of the non-surgical arm are also consistent with previous results from clinical trials (11).

We have, through our multidisciplinary team working continued to manage primary peritoneal cancer aggressively, employing chemotherapy followed by surgery in selected cases, and have shown no significant difference in stagespecific survival (20). This review confirmed no increased rate of perioperative death regardless of histology.

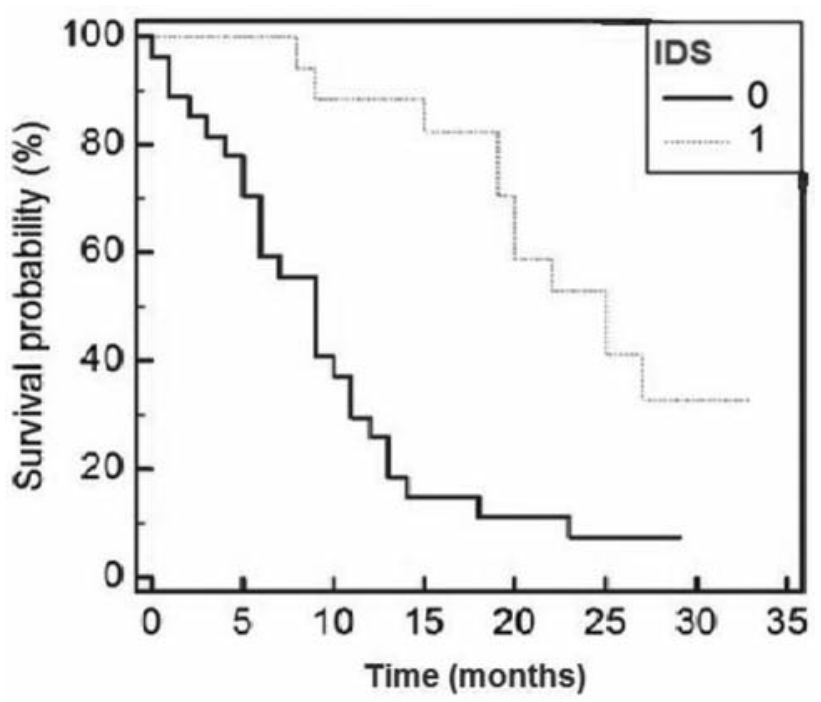

Figure 2. Kaplan-Meier curve comparing progression-free survival of surgical (1) and non-surgical (0) patients $(p=0.001)$. IDS: Interval debulking surgery.

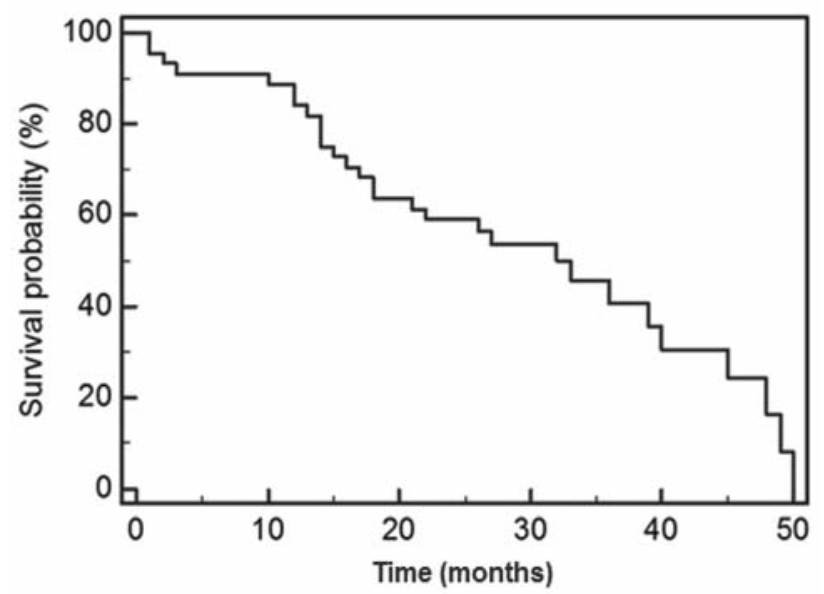

Figure 3. Kaplan-Meier curve comparing overall survival of all patients with primary peritoneal cancer $(P P C)$.

Our recommendations from this series are of consideration for interval debulking surgery (within the context of a multidisciplinary team discussion) or entry into a clinical trial for patients who have achieved a response to induction platinum-based chemotherapy.

\section{References}

1 Swerdlow M: Mesothelioma of the pelvic peritoneum resembling papillary cystadenocarcinoma of the ovary. Am J Obstet Gynecol 77: 197-200, 1959. 
2 Meinhold-Heerlein I, Fotopoulou C, Harter P, Kurzeder C, Mustea A, Wimberger P, Hauptmann S, Sehouli J; Kommission Ovar of the AGO. Statement by the Kommission Ovar of the AGO: The New FIGO and WHO Classifications of Ovarian, Fallopian Tube and Primary Peritoneal Cancer. Geburtshilfe Frauenheilkd 75(10): 1021-1027, 2015.

3 Lim D and Oliva E: Precursors and pathogenesis of ovarian carcinoma. Pathology 45(3): 229-42, 2013.

4 Kurman RJ: Origin and molecular pathogenesis of ovarian highgrade serous carcinoma. Ann Oncol 24(10): 16-21, 2013.

5 Pentheroudakis G and Pavlidis N: Serous papillary peritoneal carcinoma: unknown primary tumour, ovarian cancer counterpart or a distinct entity? A systematic review. Crit Rev Oncol Hematol 75(1): 27-42, 2010.

6 Fromm GL, Gershenson DM and Silva EG: Papillary serous carcinoma of the peritoneum. Obstet Gynecol 75: 89-95, 1990.

7 Barclay M, Gildea C, Poole J, Hirschowitz L, Menon U and Nordin A: Factors Affecting Short-term Mortality in Women With Ovarian, Tubal, or Primary Peritoneal Cancer: PopulationBased Cohort Analysis of English National Cancer Registration Data. Int J Gynecol Cancer. 2015 [Epub ahead of print]

8 Vergote I, Tropé C, Amant F, Ignace Vergote, Claes G. Tropé, Frédéric Amant, Gunnar B. Kristensen, Tom Ehlen, Nick Johnson, René H.M. Verheijen, Maria E.L. van der Burg, Angel J. Lacave, Pierluigi Benedetti Panici, Gemma G. Kenter, Antonio Casado, Cesar Mendiola, Corneel Coens, Leen Verleye, Gavin C.E. Stuart, Sergio Pecorelli and Nick S. Reed, for the European Organization for Research and Treatment of Cancer-Gynaecological Cancer Group and the NCIC Clinical Trials Group - a Gynecologic Cancer Intergroup Collaboration. Neoadjuvant Chemotherapy or Primary Surgery in Stage IIIC or IV Ovarian Cancer. N Engl J Med 363: 943-953, 2010.

9 WHO GOG criteria www.rcpath.org/resources/rcpath/ migrated $\% 20$ resources/documents/g/g079ovariandatasetnov $10 . p d f$ (accessed 17.11.15)

10 Altaras MM, Aviram R, Cohen I, Cordoba M, Weiss E and Beyth Y: Primary peritoneal papillary serous adenocarcinoma: clinical and management aspects. Gynecol Oncol 40: 230-236, 1991.

11 Ransom DT, Patel SR, Keeney GL, Malkasian GD and Edmonson JH: Papillary serous carcinoma of the peritoneum. A review of 33 cases treated with platin-based chemotherapy. Cancer 66: 1091-1094, 1990.

12 Bloss JD, Liao SY, Buller RE, Manetta A, Berman ML, McMeekin S, Bloss LP and DiSaia PJ: Extraovarian peritoneal serous papillary carcinoma: a case-control retrospective comparison to papillary adenocarcinoma of the ovary. Gynecol Oncol 50: 347-51, 1993.
13 Roh SY, Hong SH, Ko YH, Kim TH, Lee MA, Shim BY, Byun $\mathrm{JH}$, Woo IS, Kang JH, Hong YS and Lee KS: Clinical characteristics of primary peritoneal carcinoma. Cancer Res Treat 39(2): 65-68, 2007.

14 Kowalski LD, Kanbour AI, Price FV, Finkelstein SD, Christopherson WA, Seski JC, Naus GJ, Burnham JA, KanbourShakir A and Edwards RP: A case-matched molecular comparison of extraovarian versus primary ovarian adenocarcinoma. Cancer 79: 1587-1594, 1997.

15 Therasse P, Arbuck SG, Eisenhauer EA, Wanders J, Kaplan RS, Rubinstein L, Verweij J, Van Glabbeke M, van Oosterom AT, Christian MC and Gwyther SG: New guidelines to evaluate the response to treatment in solid tumors (RECIST Guidelines). J Natl Cancer Inst 92: 205-216, 2000.

16 Barda G, Menczer J, Chetrit A, Lubin F, Beck D, Piura B, Glezerman M, Modan B, Sadetzki S; National Israel Ovarian Cancer Group. Comparison between primary peritoneal and epithelial ovarian carcinoma: a population-based study. Am J Obstet Gynecol 190(4): 1039-1045, 2004.

17 Bristow RE, Tomacruz RS, Armstrong, DKTrimble EL and Montz FJ: Survival effect of maximal cytoreductive surgery for advanced ovarian cancer during the platinum era: a meta analysis. Journal of Clinical Oncology 20(5): 1248-1259, 2002.

18 The International Collaborative Ovarian Neoplasm (ICON) Group. Paclitaxel plus carboplatin versus standard chemotherapy with either single-agent carboplatin or cyclophosphamide, doxorubicin, and cisplatin in women with ovarian cancer: the ICON3 randomised trial. Lancet 360: 505-515, 2002.

19 Results from the phase II Neo-Escape trial of neoadjuvant extended sequential chemotherapy with adjuvant postoperative treatment for epithelial non-mucinous advanced inoperable peritoneal malignancy http://www.ncri.org.uk/ncriconference/ 2012abstracts/mobile/abstracts/LB15.html accessed 14/11/15.

20 Moss EL, Evans T, Pearmain P, Askew S, Singh K, Chan KK, Ganesan R and Hirschowitz L: Should All Cases of High-Grade Serous Ovarian, Tubal, and Primary Peritoneal Carcinomas Be Reclassified as Tubo-Ovarian Serous Carcinoma? Int J Gynecol Cancer 7: 1201-7, 2015
Received October 14, 2015

Revised November 18, 2015

Accepted November 30, 2015 